\title{
Digital Image Compression Enhancement Using Bipolar Backpropagation Neural Networks
}

\author{
Rafid Ahmed Khalil, Ph.D., \\ rafidamori@yahoo.com \\ University of Mosul, College of Engineering \\ Mosul, Iraq
}

\begin{abstract}
It is well known that the classic image compression techniques such as JPEG and MPEG have serious limitations at high compression rate, the decompressed image gets really fuzzy or indistinguishable. To overcome this problem, artificial neural networks ANNs techniques are used. In this paper, we propose a bipolar sigmoidal backpropagation BBP algorithm to train a feedforward autoassociative neural network. The proposed method includes steps to break down large images into smaller windows for image compression/ decompression processes. A number of experiments have been achieved, the results obtained, such as compression ratio and peak signal to noise ratio PSNR are compared with the performance of linear backpropagation LBP and standard (sigmoidal) backpropagation SBP schemes.
\end{abstract}

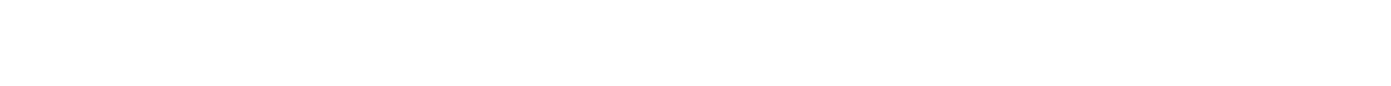 Ifin'trJF

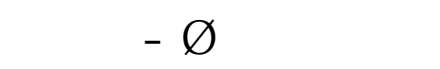

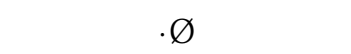

\section{ŭ}

JPEG ry̌s ¿

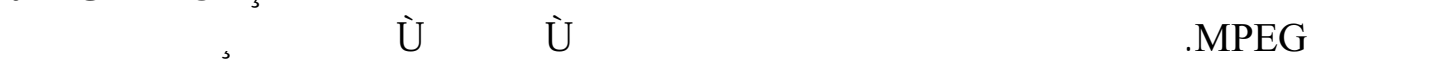

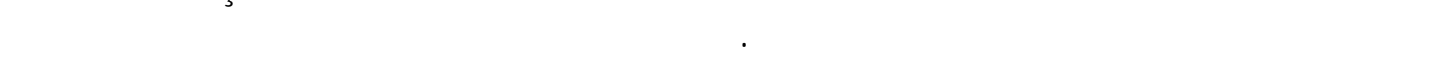

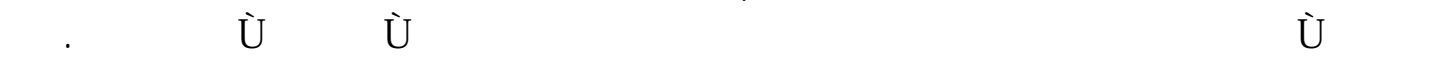

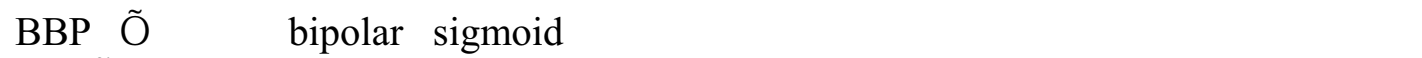

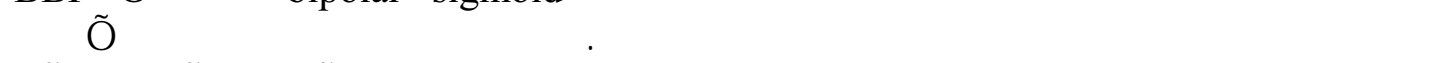

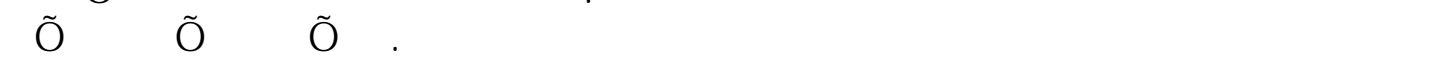

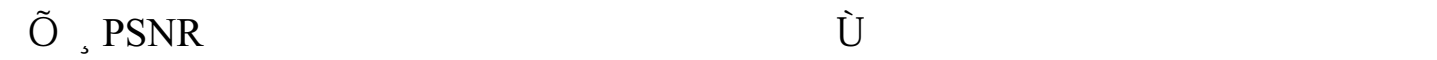

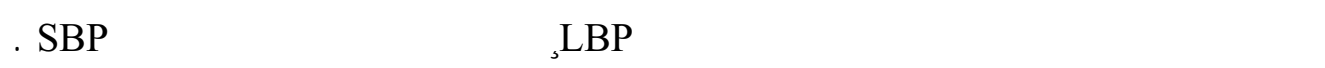




\section{$\begin{array}{llll}\text { Al-Rafidain Engineering } & \text { Vol.15 } & \text { No.4 } & 2007\end{array}$}

\section{Introduction}

Computer images are extremely data intensive, and hence require large amount of memory for storage. As a result, the transmission of an image from one machine to another can be very time consuming. By using image compression techniques, it is possible to remove some of the redundant information contained in images, requiring less storage space and less time to transmit [1].

Artificial neural networks ANNs have been applied to image compression problems, have demonstrated their superiority over traditional methods, when providing high compression rate, and high signal to noise ratio [2].

Several literatures were discussed the subject of applying ANNs to image compression in detail as in $[1,3,5]$. Many different training algorithms and architectures have been used. Some of the most used algorithms are standard backpropagation [5], linear backpropagation [4- 6], and other advanced schemes of backpropagation neural networks [7, 8]. Self- organizing maps SOMs neural network, which after training acts as a codebook [9]. ART network used for adaptive fussy approach [10], and radial basis function RBF neural network [11].

The purpose of this paper is to present a technique for compression of images using bipolar sigmoidal nonlinear function with backpropagation algorithm BBP, applied to train 2-layer feedforward autoassociative neural network. Results are presented for BBP, and compared for both standard (sigmoidal) backpropagation SBP, and Linear backpropagation LBP. The comparison includes compression rate, and the peak signal to noise ratio PSNR (the quality of decompressed image).

This paper is organized as follows: In section 2, we describe the network architecture and activation functions for backpropagation. The image compression procedure is presented in section 3. Performance assessments are presented in section 4. In section 5, a performance comparison among backpropagation schemes is performed by the help of some experimental results. Section 6 is dedicated to conclusions.

\section{The Neural Network}

\subsection{Architecture}

The Backpropagation (BP) is one of neural networks, which are directly applied to image compression. The network structure is illustrated in Figure (1). This structure referred to feedforward autoassociative type network. The input layer and output layer are fully connected to the hidden layer. Compression is achieved by estimating the value of $K$, the number of neurons at the hidden layer less than that of neurons at both input and the output layers. 


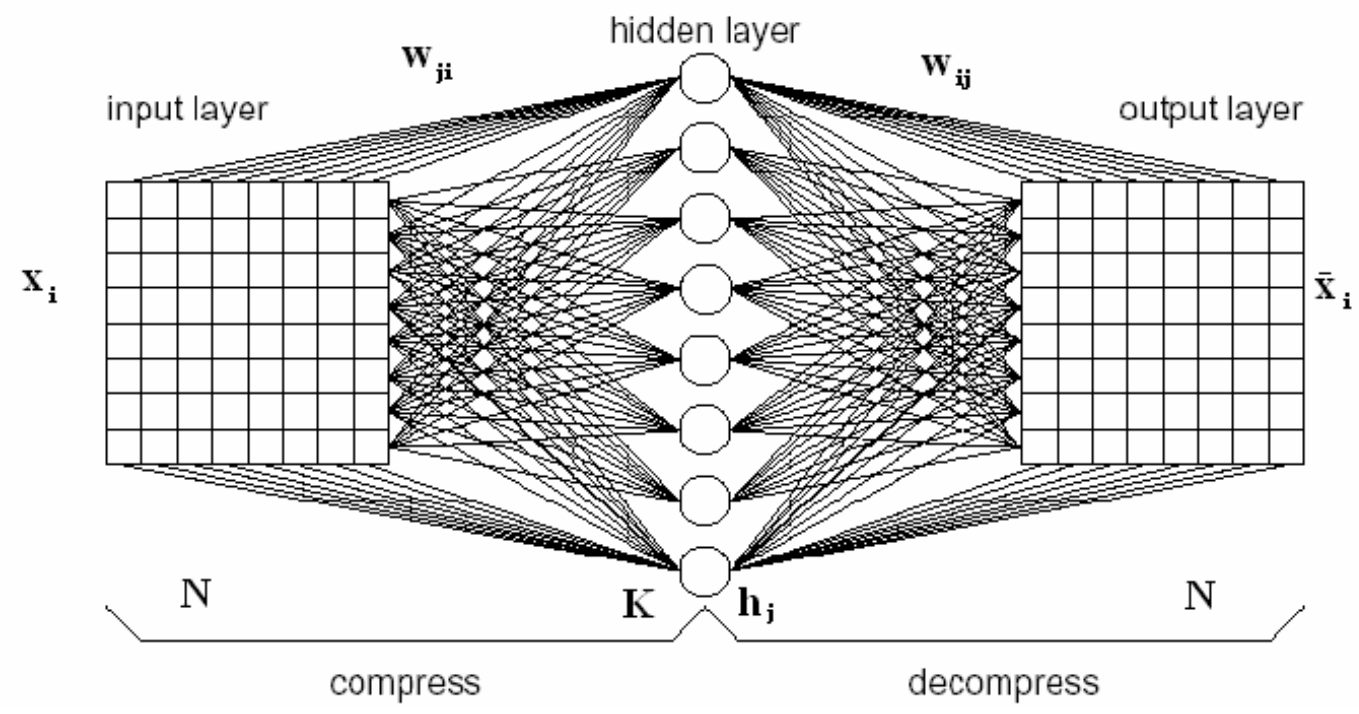

Figure 1. The N-K-N neural network. There are actually connections between each of the $\mathrm{N}$ neurons in input or output layer and the $\mathrm{K}$ neurons of hidden layer.

The input image is split up into a number of blocks, each block has $N$ pixels, which is equal to the number of input neurons see Figure (2) [6].

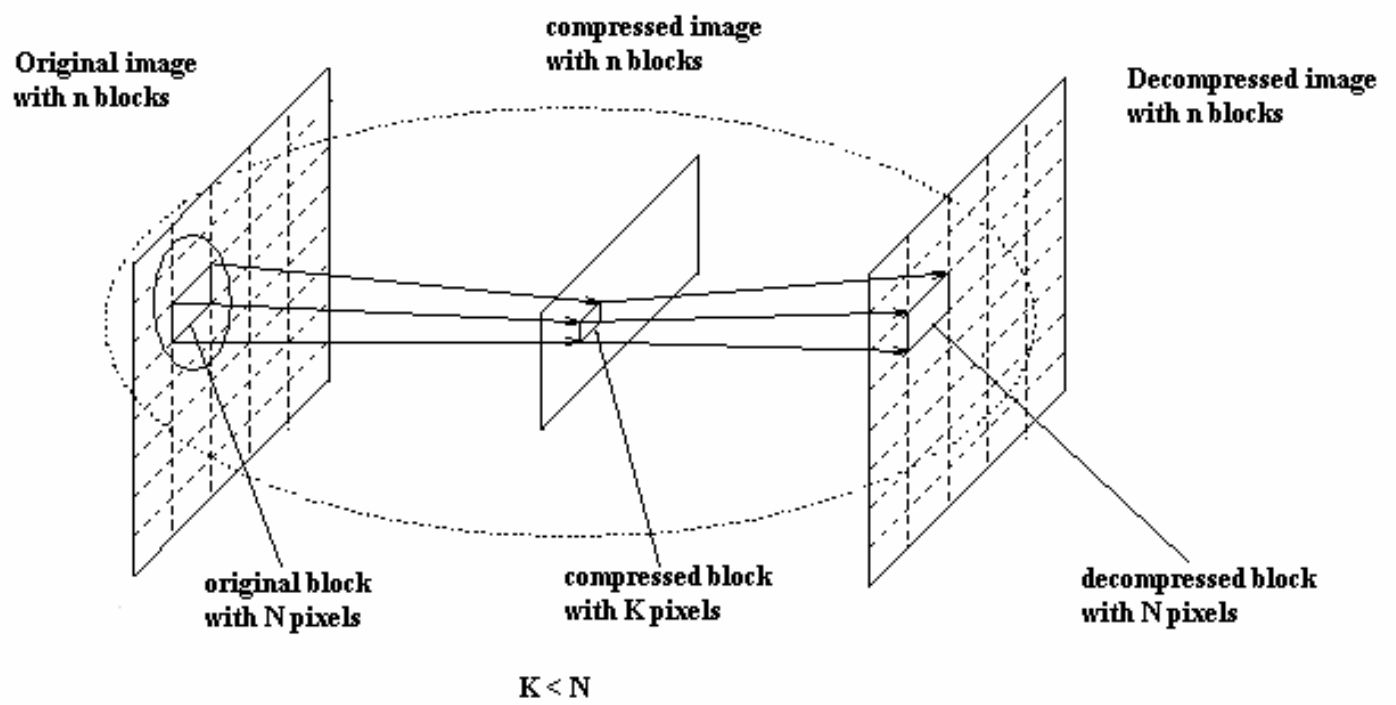

Figure 2. Compression of an arbitrarily large image using a neural compressor/ decompressor . 


\subsection{Activation function}

In accordance with the neural network structure shown in Figure (1), the operation of BP algorithm can be described as follows[12]:

$$
h_{j}=f\left(\sum_{i=1}^{N} w_{j i} x_{i}\right), \quad 1 \leq j \leq K
$$

For compression

$$
\bar{x}_{i}=f\left(\sum_{j=1}^{K} w_{i j}^{\prime} h_{j}\right), \quad 1 \leq i \leq N
$$

For decompression

Where $\left\{w_{j i}, j=1,2, \ldots \ldots, K\right.$ and $\left.i=1,2, \ldots ., N\right\}$, the connection weights from input layer to hidden layer, and $\left\{w_{i j}^{\prime}, i=1,2, \ldots . N\right.$ and $\left.j=1,2, \ldots . K\right\}$, the connection weights from hidden layer to output layer.

$x, \in(-1.0,1.0)$ denotes the normalized pixel values for grey scale images with grey levels $(0-255) . f($.$) is a nonlinear activation function. This function is$ sigmoidal for standard backpropagation SBP algorithm, and can be written as:

$$
f(u)=\frac{1}{1+e^{-\beta x}}
$$

This function is continuous and varies from 0.0 to 1.0 as $u$ varies from $-\infty$ to $+\infty$. The gain of the sigmoid $\beta$, determines the steepness of transition region of the function [13].

In this paper, we propose to use bipolar sigmoidal nonlinear function with backpropagation $\mathrm{BBP}$, and are given by:

$$
f(u)=\frac{1-e^{-\beta x}}{1+e^{-\beta x}}
$$

This function is also a continuous function and varies from -1.0 to +1.0 as $u$ varies from $-\infty$ to $+\infty$. For a linear backpropagation LBP, the activation function is given as:

$$
f(u)=\frac{u}{\left|u_{\max }\right|}
$$

Where $\left|u_{\max }\right|$ is a maximum absolute value of $u$. 


\section{Image Compression Scheme}

The network used for image compression is breaked into two parts as shown in Figure (3). The transmitter encodes and then transmits the output of hidden layer ( only $K$ values as compared to $N$ of the original image). The receiver receives and decodes the $K$ hidden outputs and generates the $N$ outputs. Since the network is implementing an identity map, the reconstruction of the original image is acheived [14].

In order to get a higher compression, we quantized the hidden layer output into 8 or 16 distinct level, coded on 3 or 4 bits. In that way, the original $N$ bytes are compressed to $\frac{3 K}{8}$ or $\frac{4 K}{8}$ bytes and getting a high compression rate. To decompress a compressed image, the output layer of the network is used. The binary data is decoded back to real number and propagated through the $N$ neurons layer to output, then converted from range $(-1.0,+1.0)$ to positive integer values between 0 and 255 ( grey level).
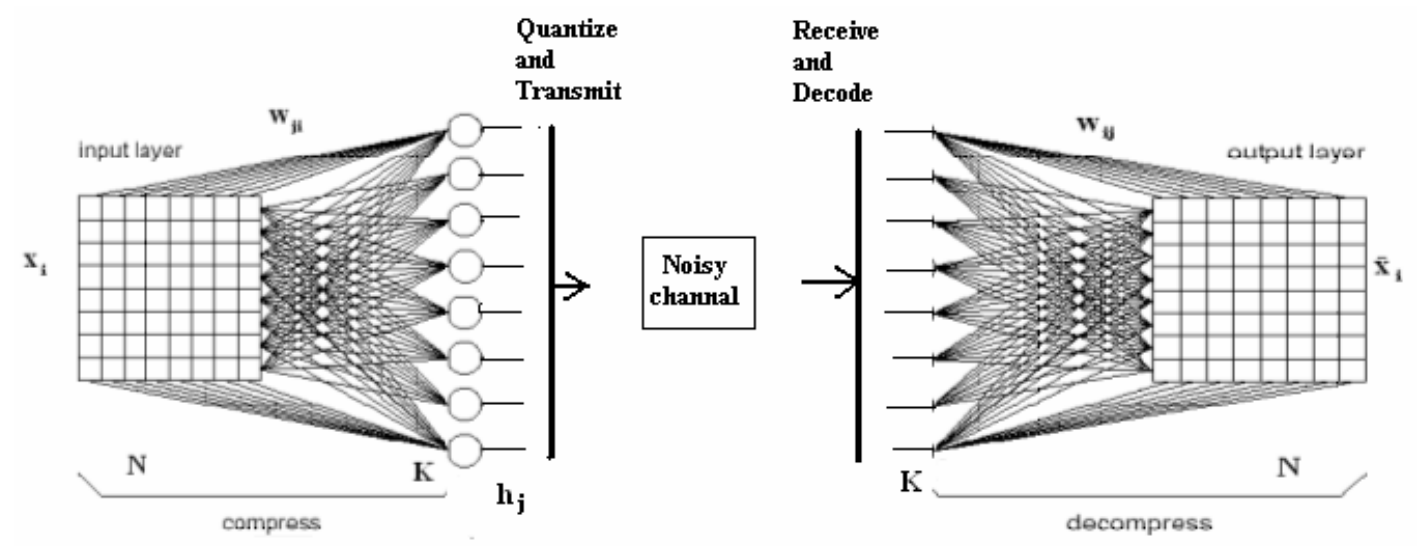

Figure 3. A neural network compression/ decompression pair.

\section{Performance assessments}

Several significant images were chosen to train the neural networks, each of those images had ( 256 X 256) pixels, and splited up into ( 32 X 32) blocks, every block consist of ( 8 X 8) pixels (eight bits per pixel). Each block fed to ( $64-16-64)$ neural network in random order see Figure (4a). Once the network was trained on a specific image, it performed very well on virtually any other different image see Figure $(4 \mathrm{~b}$ and $\mathrm{c})$. 


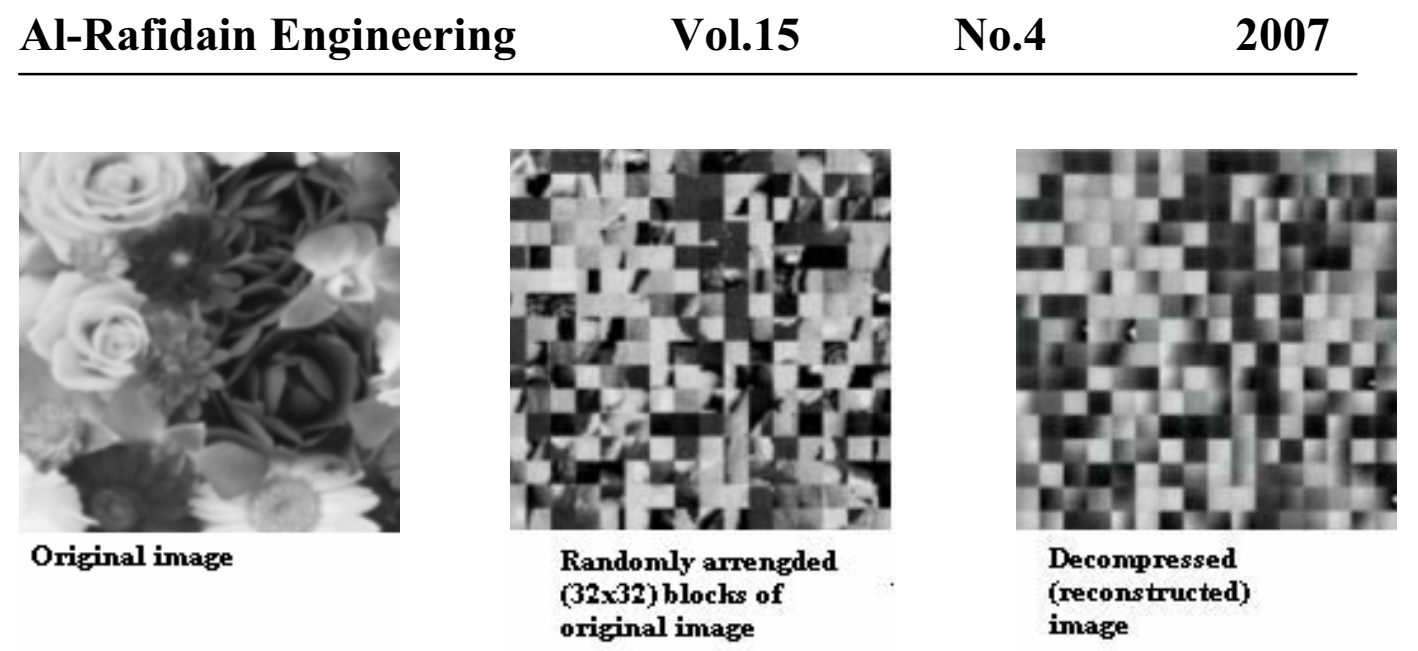

(a) Neural network - learning phase.

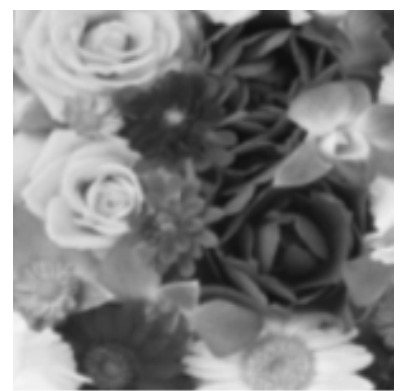

Original image

input layer

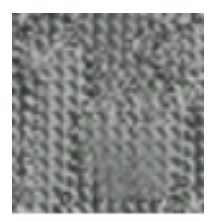

Compressed

image

hidden layer

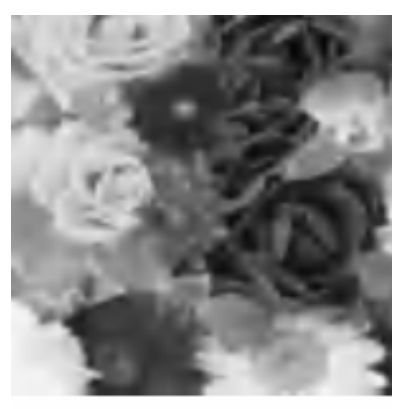

Decompressed image output layer

(b) (b) Neural network - recall phase.

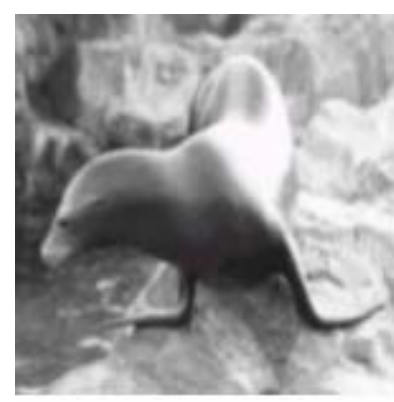

Different original image input layer

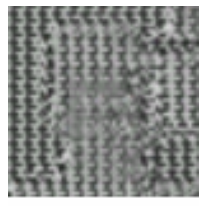

Compressed image hidden layer

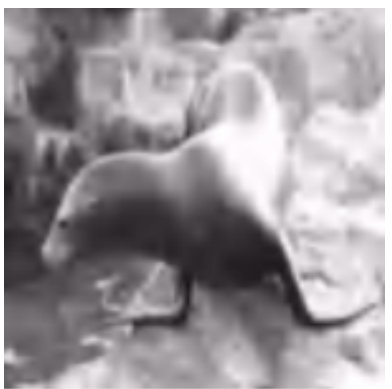

Decompressed image output layer

(c) Neural network - generalization test.

Figure (4) Learning, testing processes for compression/ decompression of image within the artificial neural network. 
The performance of the three backpropagation schemes BBP, LBP, and SBP can be assessed by considering the following measurements:

a. Compression Ratio ( $\mathfrak{R}$ ) [15], and can be defined for a narrow channel compression neural network as follows:

$$
\mathfrak{R} \equiv N: \frac{T K}{8}
$$

where $T$ is the number of bits used to quantize each hidden neuron output.

b. Peak Signal to Noise Ratio PSNR [5], is the measure for the quality of reconstructed or decompressed image, and can be define for 256- gray level image with $n$ blocks of size $N$ pixels as follows:

$$
P S N R=10 \log \frac{(256-1)^{2}}{\frac{1}{n N} \sum_{i=1}^{n} \sum_{j=1}^{N}\left(\bar{P}_{i j}-P_{i j}\right)^{2}}, d B
$$

where $\bar{P}_{i j}$ is the intensity value of pixels in the reconstructed images;

in addition, $P_{i j}$ is the intensity value of pixels in the original images, which are split up into $n$ inputs (blocks): and,

$$
\begin{aligned}
& x_{i}=\left\{P_{i 1}, P_{i 2}, \ldots \ldots \ldots, P_{i N}\right\} \\
& \bar{x}_{i}=\left\{\bar{P}_{i 1}, \bar{P}_{i 2}, \ldots \ldots \ldots . ., \bar{P}_{i N}\right\}
\end{aligned}
$$

\section{Experimental Results and Discussions}

To test and compare the compression performance of the proposed BBP scheme with SBP and LBP schemes, several images are used in the learning phase as shown in Fig (5). Then we take Barbara as one of most widely used image for testing image compression algorithms. Table $(1-3)$ summaries the experiments results, and Figure ( $6-7$ ) show the compression performance for the backpropagation schemes for different neural network parameters. 


\begin{tabular}{llll} 
Al-Rafidain Engineering & Vol.15 & No.4 & 2007 \\
\hline
\end{tabular}

Table 1.Standard backpropagation SPB on Lenna image of (256x256) pixels.

\begin{tabular}{|c|c|c|c|c|}
\hline $\begin{array}{c}\text { Dimension } \\
\mathrm{N}\end{array}$ & $\begin{array}{c}\text { Quantization } \\
\text { level }\end{array}$ & $\begin{array}{c}\text { Hidden } \\
\text { neuron K }\end{array}$ & Compression rate & PSNR, dB \\
\hline 64 & $2^{4}$ & 16 & $8: 1$ & 21.4 \\
\hline 64 & $2^{3}$ & 16 & $16: 1$ & 20.0 \\
\hline 64 & $2^{4}$ & 8 & $10.5: 1$ & 20.6 \\
\hline 64 & $2^{3}$ & 8 & $21: 1$ & 18.7 \\
\hline
\end{tabular}

Table 2. Linear backpropagation LPB on Lenna image of (256x256) pixels.

\begin{tabular}{|c|c|c|c|c|}
\hline $\begin{array}{c}\text { Dimension } \\
\mathrm{N}\end{array}$ & $\begin{array}{c}\text { Quantization } \\
\text { level }\end{array}$ & $\begin{array}{c}\text { Hidden } \\
\text { neuron K }\end{array}$ & Compression rate & PSNR, dB \\
\hline 64 & $2^{4}$ & 16 & $8: 1$ & 24.2 \\
\hline 64 & $2^{3}$ & 16 & $16: 1$ & 21.2 \\
\hline 64 & $2^{4}$ & 8 & $10.5: 1$ & 21.5 \\
\hline 64 & $2^{3}$ & 8 & $21: 1$ & 20.1 \\
\hline
\end{tabular}

Table 3.Bipolar sigmoidal backpropagation PPB on Lenna image of (256x256) pixels .

\begin{tabular}{|c|c|c|c|c|}
\hline $\begin{array}{c}\text { Dimension } \\
\mathrm{N}\end{array}$ & $\begin{array}{c}\text { Quantization } \\
\text { level }\end{array}$ & $\begin{array}{c}\text { Hidden } \\
\text { neuron K }\end{array}$ & Compression rate & PSNR, dB \\
\hline 64 & $2^{4}$ & 16 & $8: 1$ & 29.0 \\
\hline 64 & $2^{3}$ & 16 & $16: 1$ & 25.4 \\
\hline 64 & $2^{4}$ & 8 & $10.5: 1$ & 27.1 \\
\hline 64 & $2^{3}$ & 8 & $21: 1$ & 24.5 \\
\hline
\end{tabular}




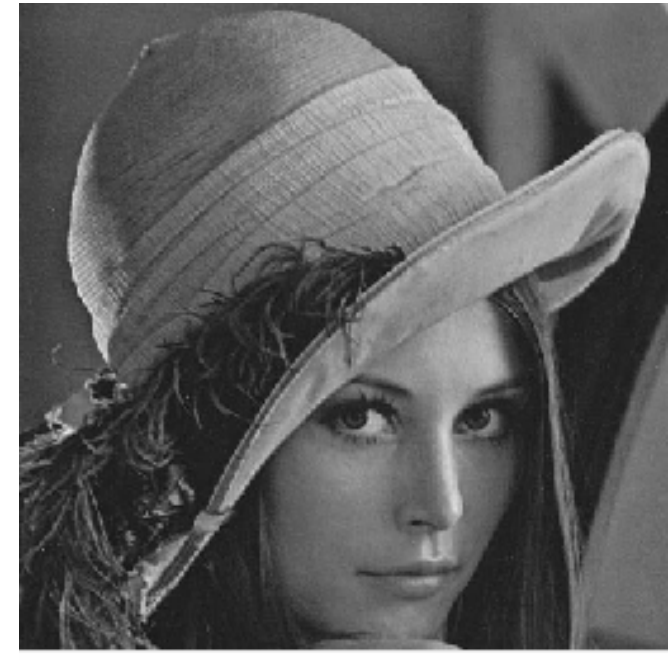

Original image $256 \times 256$ pixels

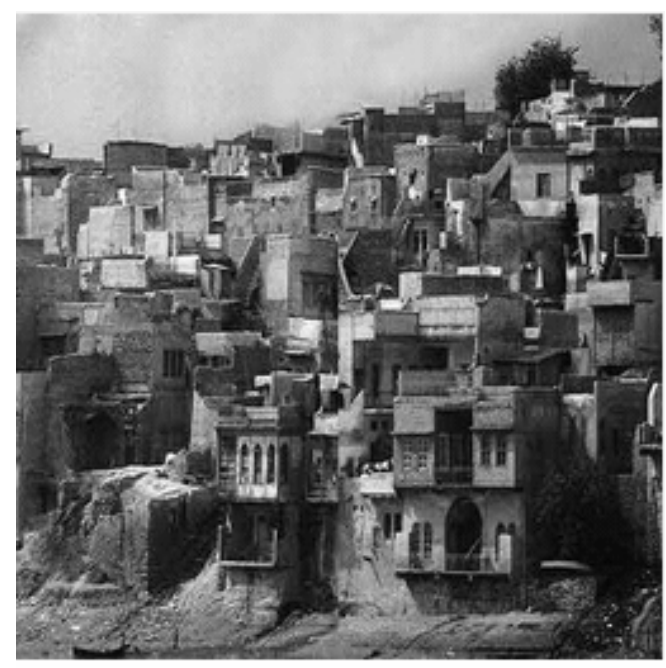

Mosul old houses original image $256 \times 256$ pixels

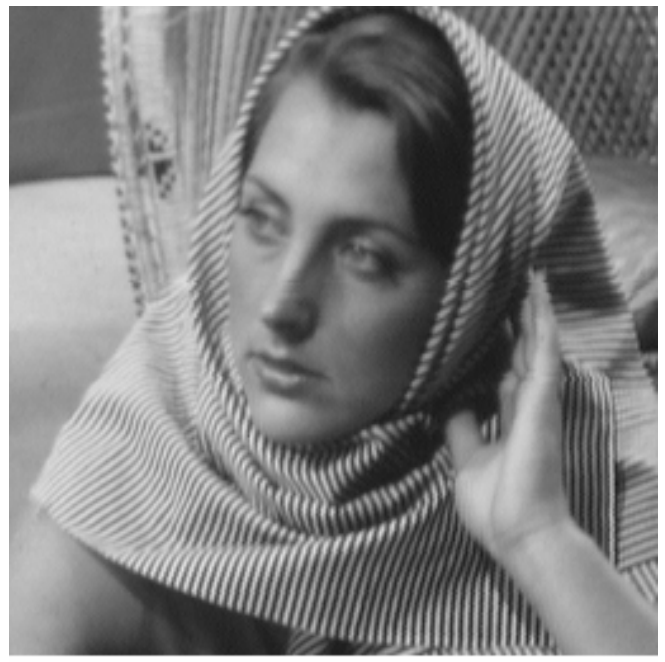

Barbara Original image $256 \times 256$ pixels

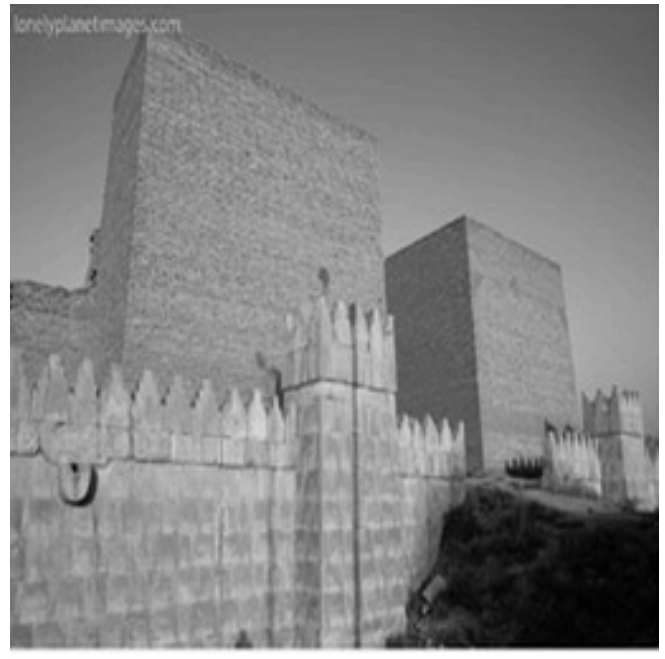

Wall \& gate of the ancient city of Nineveh, original image $256 \times 256$ pixels

Figure (5) Four different images used in learning phase of the neural network. 


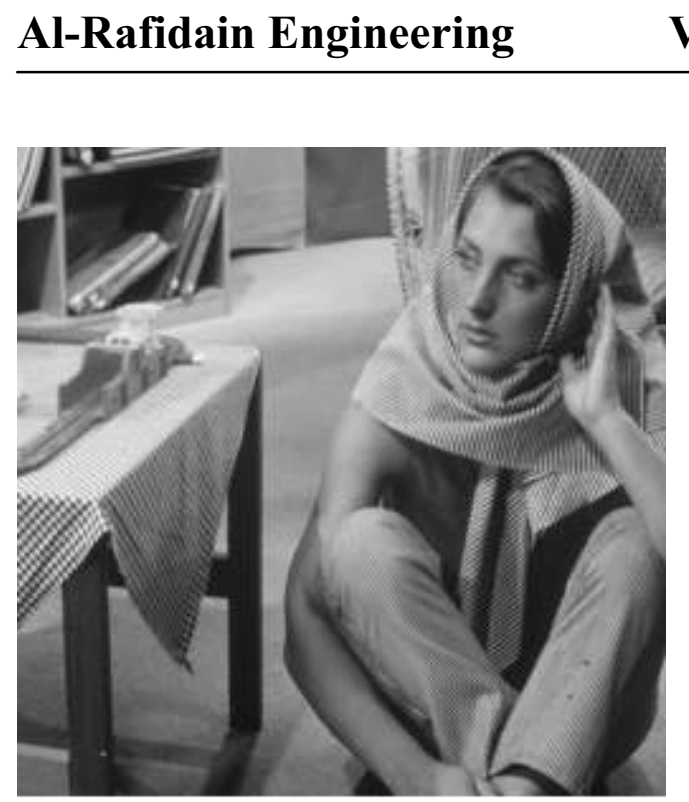

Original Image

$256 \times 256$ pixels

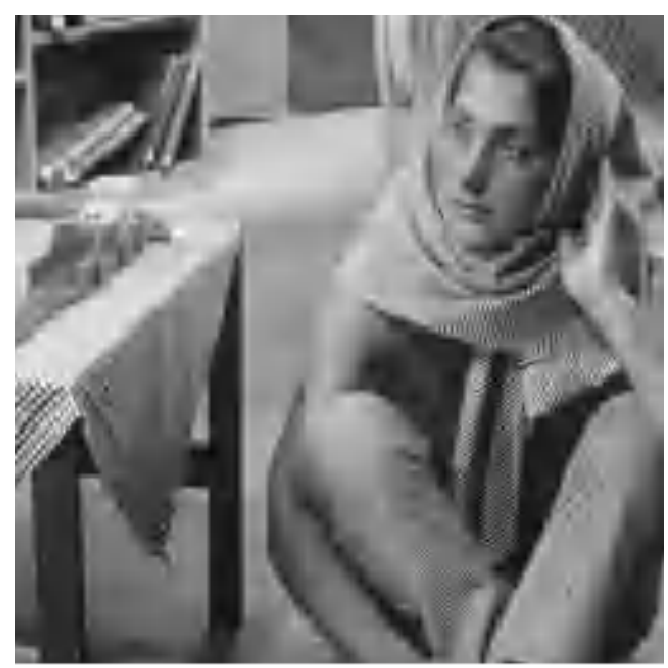

Using LPB

21: $1, P S N R=20.6 \mathrm{~dB}$
Vol.15 No.4

2007

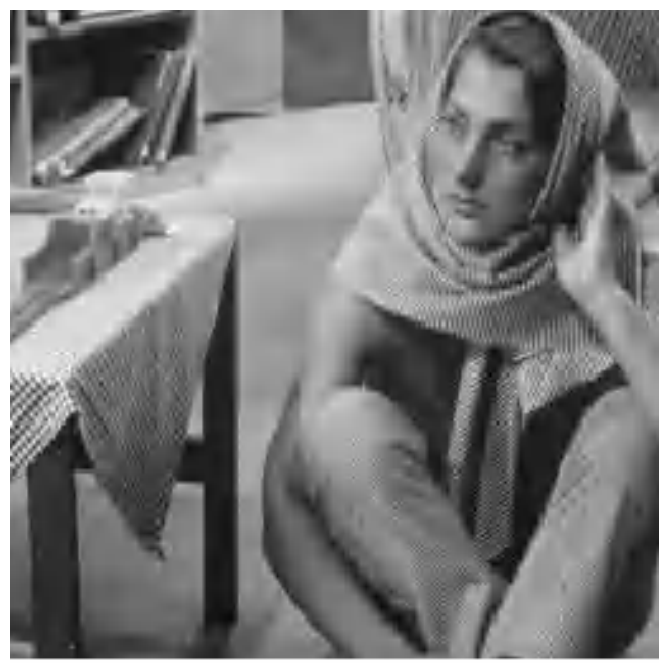

Using BBP

21: 1, PSNR $=25.3 \mathrm{~dB}$

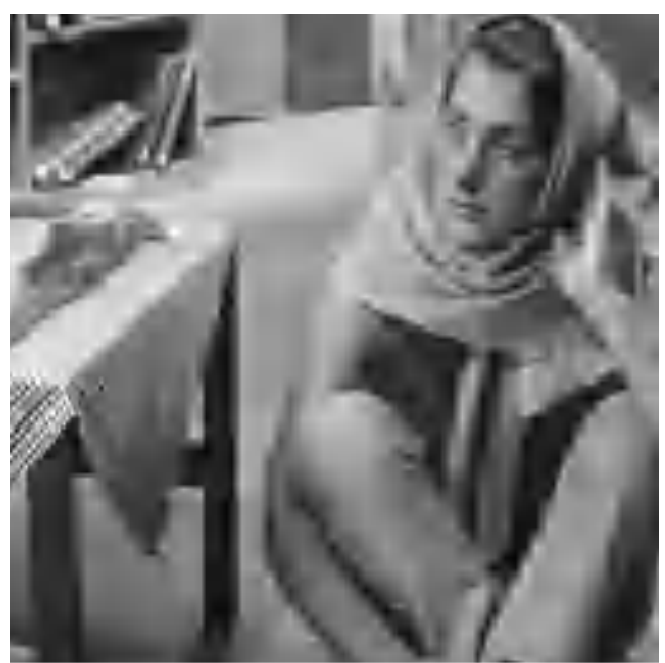

Using SBP

$21: 1, P S N R=19.1 \mathrm{~dB}$

Figure (6) Testing results for 21:1 compression rate with 64-16-64 neural network. 


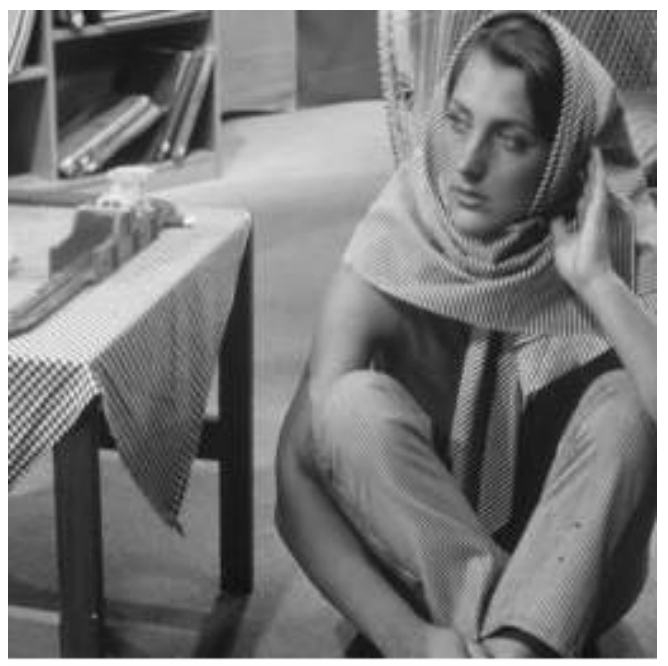

Original Image $256 \times 256$ pixels

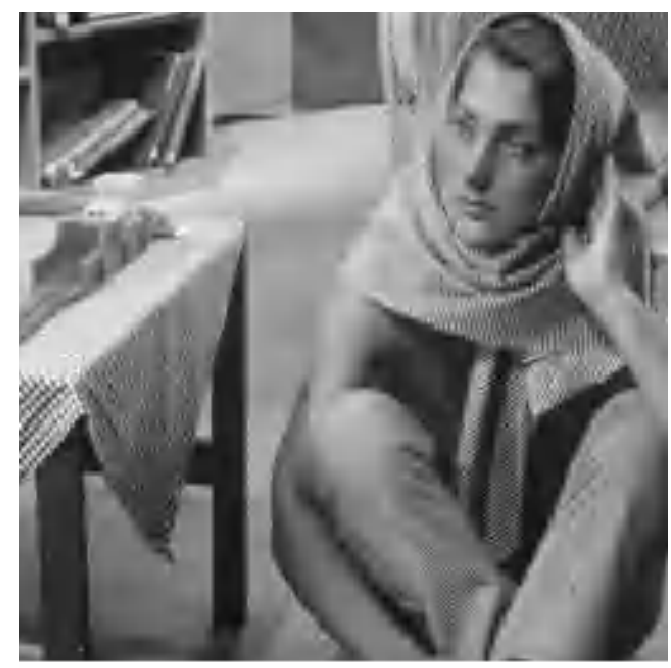

Using LBP

$8: 1, P S N R=24.9 \mathrm{~dB}$

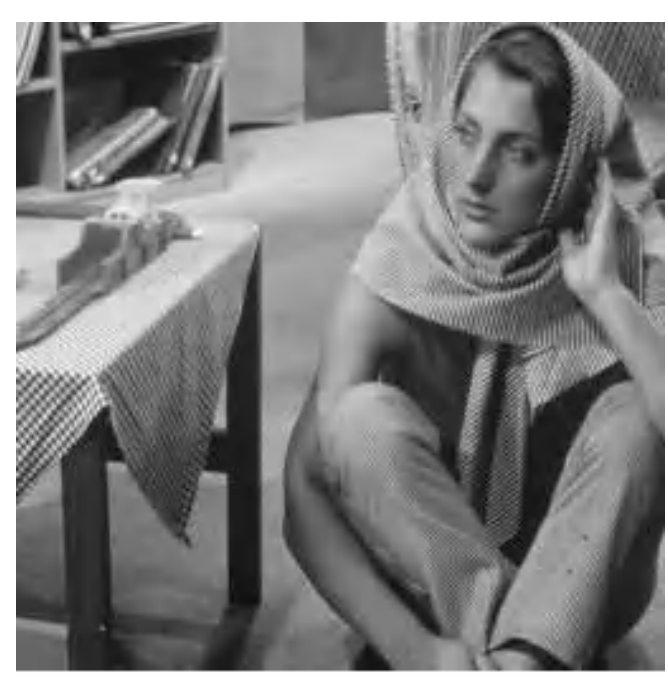

Using BBP

8: $1, P S N R=29.7 \mathrm{~dB}$

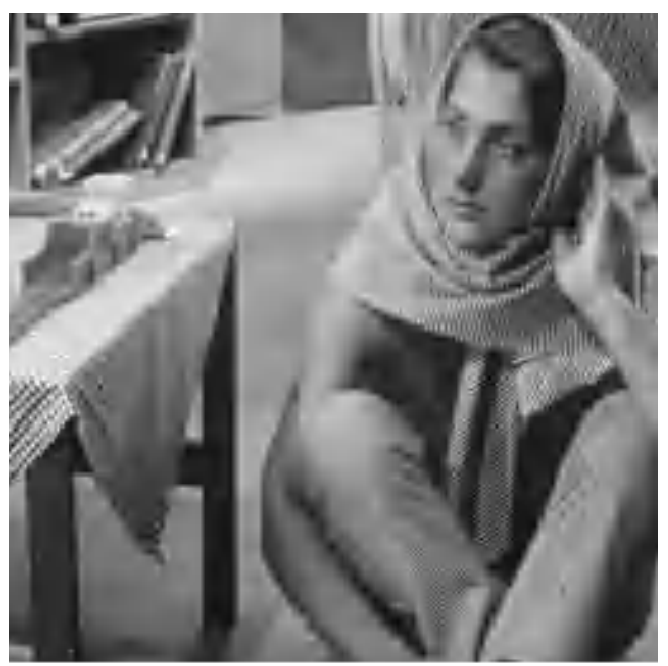

Using SBP

$8: 1, P S N R=22.1 \mathrm{~dB}$

Figure (7) Testing results for 8:1 compression rate with 64-8-64 neural network. 


$\begin{array}{llll}\text { Al-Rafidain Engineering } & \text { Vol.15 } & \text { No.4 } & 2007\end{array}$

As can be seen from Tables ( $1-3$ ), the three backpropagation schemes performed quite well. The compression rate in the three tables reflected how accurately the images were decompressed. The three schemes showed very little deterioration of the image quality when using an appropriate number of neurons in the hidden layer of the neural networks. As the number of hidden neurons was lowered, the greater deterioration in the decompressed images. However, the lower the number of hidden neurons, the better the compression ratio of the image.

The images results from testing compression / decompression capabilities and performance of there backpropagation schemes are shown in Figure ( 6, 7). All images suffered little in quality but no deterioration. A higher peak signal to noise ratio PSNR of order of $30 \mathrm{~dB}$ was achieved for BBP scheme, and lower PSNR of value $19 \mathrm{~dB}$ for SBP scheme, for the same neural network parameters. Overall, it was found that superior performance could be achieved with the proposed BBP scheme, while the SBP shows inferior performance.

\section{Conclusions}

This paper has successfully applied bipolar sigmoidal backpropagation BBP to a large, complex task. The results appear to be promising in image compression/ decompression problems. It is used to train a feedforward autoassociative network. We segmented, compressed, decompressed, and reconstructed various images using this method. A number of experiments have been conducted. Results showed that a superior PSNR could be achieved with proposed BBP compared with SBP and LBP.

\section{References}

1. M. Egmont-Ptersen, D. de Ridder, H. Handels, "Image processing with neural networks- a review", Pattern Recognition , 35 (2002) pp. 2279- 2301.

2. B. Verma, M. Blumenstein, S. Kulkarni, "A new compression technique using an artificial neural network". Faculty of Information and Communication Technology, Griffith University, Australia, 2004. \{URL:http://citeseer.ist.psu.edu/update/29252

3. "Digital Imaging \& Data compression; Image compression with neural networks". \{URL:http://www.comp.glam.ac.uk.digimaging/neural.htm/\}

4. R. D. Dony, S. Haykin, "Neural network approaches to image compression", Proc. IEEE 83 (2) (February 1995) pp. 288- 303.

5. J. Jiang, "Image compression with neural networks - A survey, Signal Processing": Image Communication 14 (1999) pp. 737 - 760. 
6. E. Gelenbe, M. Sungur, C. Cramer, "Learning random networks for compression of still and moving images", Dept. Ele. Engg., Duke University, 2003. $\{$ URL:http://www.ee.duke.edu./ cec/research/neuralcom pression/JPL/paper.html $\}$.

7. Md. I. Bhuiyan, Md. K. Hassan, "Image compression with neural network using DC algorithm", Journal of signal processing, vol. 5, no. 6, (2001) pp. $445-459$.

8. A. K. Ashikur, C. Mofizur, "A new approach for compressing images using neural network", CIMCA Proceeding, (February 2003), Vienna - Austria.

9. C. Amerijckx, M. Verleysen, "Image compression by self- organized Kohenon map, IEEE Trans. Neural Networks", 9 (3), (1998), pp. 503 - 507.

10. S. Mitra, S. Yang, "High fidelity adaptive vector quantization at very low bit rate for progressive transmission images", J. Electron. Imaging, 8(1), (1999), pp. 23- 35 .

11. S. Rizvi, L. Wang," Nonlinear vector prediction using feedforward neural network", IEEE Trans. Image Process., 6(10), (1997), pp. 1431- 1436.

12. D. Hush, and B. Horne, "Progress in Supervised neural networks". IEEE Signal processing Magazine, (January 1993), pp. 8- 39.

13. D. Rumelhart, G. Hinton, R. Williams, "Learning internal representations by error propagation", in Parallel Distributed Processing, MIT Press, Vol. 1, (1986), pp. 318-362.

14. M. S. Rahim, "Image compression by new sub- image block classification techniques using neural network. IEICE Trans. on Fundamentals of Electronics, Communications, and Computer Sciences, E83-A (10), (2000), pp. 2040- 2043.

15. R. Jigrea, L. Pajtek, " Neural networks image compression: a way to real time movie transmission over slow serial lines, Automation and Computer Science Faculty, University of Timisora, Romania, (2004). \{URL:http://citeseer.ist.psu.edu/correct/975252\}.

The work was carried out at the college of Engg. University of Mosul 